\title{
Processing of Porous Ceramics by Direct Foaming: A Review
}

\author{
Ashish Pokhrel, Dong Nam Seo, Seung Taek Lee, and Ik Jin Kim ${ }^{\dagger}$ \\ Institute of Processing and Application of Inorganic Materials (PAIM), \\ Department of Materials Science and Engineering, Hanseo University, Chungnam 356-706, Korea
}

(Received September 19, 2012; Revised November 16, 2012; January 29, 2013; Accepted March 14, 2013)

\begin{abstract}
Macro porous ceramics possessing controlled microstructures and chemical compositions have increasingly proven useful in the industrial sphere. Their sintered structures have found application in both established and emerging, areas such as thermal insulation in buildings, filtration of liquids and molten materials, refractory insulation, bone scaffolds and tissue engineering. Stable ceramic foams can be formed by wet chemical methods using inorganic particles(e.g., $\mathrm{Al}_{2} \mathrm{O}_{3}$ or $\mathrm{SiO}_{2}$ ). The wet foams are dried and sintered with improved porosity and mechanical properties. This review examines the different techniques used to prepare porous ceramics from ceramic foams, focusing on the explanation of this versatile method of direct foaming from the past to the present. Comparisons of the processes and the processing parameters are explained with the produced microstructures.
\end{abstract}

Key words : Porous ceramics, Direct foaming, In-situ hydrophobization, Wet foam stability

\section{Introduction}

$\mathbf{P}$ orous ceramics have been widely used in various versatile applications, such as liquid gas filters, catalysis supports, gas distributors, insulators, preforms for metalimpregnated ceramic metal composites and implantable bone scaffolds. ${ }^{1)}$ Unlike in metallic or polymeric products, pores have traditionally been avoided in ceramic components because of their inherently brittle nature. ${ }^{2,3)}$ However, porous ceramics have become increasingly essential, especially for use in environments involving high temperatures, extensive wear and corrosive media. ${ }^{4)}$ Porous ceramics are specially advantageous in biological applications because of their high melting point, tailored electronic properties, high resistance to corrosion and surface damage. When porous ceramics are synthesized using favorable conditions it provides suitable features by introducing voids into the solid structures. These features include low thermal conductivity, controlled permeability, high surface area, low density, high specific strength and low dielectric constant.5) These properties can be tailored for each specific application by controlling the composition and microstructure of the porous ceramics. Changes in open and closed porosity pore size distribution and pore morphology can greatly affect a material's properties. These microstructural features are highly influenced by the processing route used to produce the porous material. ${ }^{2)}$ Various prominent discoveries have been done over the last few decades in the field of porous ceramics and its processing, but in this review we have focused

\footnotetext{
${ }^{\dagger}$ Corresponding author : Ik Jin Kim

E-mail : ijkim@hanseo.ac.kr

Tel : +82-41-660-1441 Fax : +82-41-660-1402
}

specifically on the direct foaming process of synthesizing porous ceramics.

\subsection{Direct Foaming (past to present)}

First, the direct foaming of pre-ceramic polymer mixtures, followed by high temperature pyrolysis in an inert atmosphere, have been employed to fabricate ceramic foams with impressive strength, stiffness, thermo mechanical and thermochemical durability, and electromagnetic properties. ${ }^{6)}$ This approach involved mixing a pre-ceramic polymer (usually a silicone resin) with precursors for polyurethane (polyols and isocyanates) in a common solvent, which also acted as a physical foaming agent for the system. Then foam was blown outwards by vigorous stirring of the mixture and inserting the sample into an oven previously kept at a controlled temperature. Moreover, different novel processing methods for cellular ceramics, including the burning out of fugitive pore formers and chemical processing by templates, have been proposed in studies of micro- cellular ceramics and pre-ceramic polymers. ${ }^{7}$ Similar processing methods have been described in other literary studies regarding cellular ceramic structures that were conventionally produced by dip-coating polymeric foam into ceramic slurry, followed by burnout of the preform and sintering. ${ }^{8)}$ This approach led to the production of parts with high porosity and low cost but limited strength, which are suitable for molten filters or kiln furniture. Furthermore, each fabrication method was best suited for producing a specific range of cell sizes, cell size distribution and the overall amount of porosity, as well as for influencing the level of interconnectivity among the cells, amount, thickness and orientation of the cell walls. The thickness of the struts was also influenced by the fabrication procedure, and its parameters, together with the rel- 
ative density of the materials, directly affects the strength of the components. ${ }^{9)}$ The studies introduced by Colombo et al. on direct foaming gave insights on the generation of bubbles inside liquid slurry containing ceramic powders or inside a ceramic precursor solution to create foam that then needed to be set in order to maintain its porous morphology before being heated to high temperature for sintering. The nucleation of the gas bubbles inside the liquid slurry is influenced by the presence of suspended particles, and the bubbles that initially had a spherical shape later grew into polyhedral cells. Thus, the processes were demonstrated through judicious selection of the pre-ceramic polymer, manipulation of the foaming and pyrolysis processes.

Focusing on all these vital parameters, the individual research of other pioneers on porous ceramics explained the importance of the porous ceramics in the present day and dealt with discoveries by various scientists. P. Greil and his associates proposed advanced concepts for ceramics engineering to fulfill the requirements of increased precision in both design and manufacturing to achieve greater uniformity and predictability in the materials performance. ${ }^{10)}$ They discovered that the way of preparing pre-ceramic foam to produce porous ceramics is by direct foaming using a thermal pre-curing of filler-loaded poly (silesquioxane) on the foam structure formation by an in-situ foaming reaction. Magnesium alloy-ceramic foam composite materials (IPCs) were produced by infiltrating the open cell polymer-derived ceramic foams by squeeze casting. ${ }^{11)} \mathrm{A}$ foaming agent was used for generating the foam structure which is formed by the condensation reaction of the hydroxy and ethoxy groups with silanol groups of the phenyl methyl poly (silesesquioxane) at temperatures above $200^{\circ} \mathrm{C} .^{11)}$ Not only were the cellular foams formed by direct foaming but also the high utility metallic foams were explained by Banhart and his work associates. ${ }^{12)}$ They proposed supersaturated metal-gas systems under high pressure that initiate bubble formation by pressure and temperature control. Their studies focused on the liquid-gas mixtures solidified to form solid foam and other metal foams such as $\mathrm{TiH}_{2}, \mathrm{ZrH}_{2}$, and $\mathrm{MgH}_{2}$ by hydrogen desorption. ${ }^{13,14)}$ These were performed with aluminum and zinc alloys with the disadvantage of the foaming agent releasing gas far below the melting point of the metal, which will expand in the solid state and yield crack-like pores and heterogeneous pore structures. If the foaming agent decomposed far above the melting point, the viscosity of the melt would be too low to allow for the formation of stable foam. ${ }^{15,16)}$ Researchers from Nottingham University gave some interesting insights on the direct foaming process for obtaining porous ceramics. Hydroxyapatite foams were produced in aqueous suspension with agar, a polysaccharides preparation by refrigeration. The foams were generated by mechanical incorporation of air or by injection of gas. ${ }^{16)}$ Among the alternative foaming agents iota carrageenan gum (iCG) from red sea weed that gel in aqueous solution on heating, stable ceramic foams were formed by the mechanical frothing of the mixture of the solution. Another author also summarized the processes of manufacturing the porous ceramics from aqueous foams. The ceramic manufacturing processes were classified in such a way that the connections to related operations in other industries and to the ancient crafts of antiquity became apparent. ${ }^{17)}$ Another innovative processing route for developing microcellular ceramics was developed as discussed in the literature. ${ }^{18)}$ In this method the technology relied on the implementation of the principle of inducing a thermodynamic instability in the production process for microcellular pre-ceramic polymers that further transformed into microcellular ceramics using pyrolysis. The main strategies were i) saturating pre-ceramic polymers using gaseous, liquid, or supercritical $\mathrm{CO}_{2}$; ii) nucleating and growing a large number of bubbles using thermodynamic instability via a rapid pressure drop and or heating; and iii) transforming the microcellular pre-ceramic polymers into microcellular ceramics by pyrolysis and optional subsequent sintering. During pyrolysis in an inert atmosphere, the polymer-to-ceramic transformation of the pre-ceramic occurred, yielding a silicon carbide for PCS or a silicon oxy-carbide ceramic for polysiloxane. Avoiding the collapse of the pore structure is the primary concern during the pyrolysis and sintering processes. It has been highlighted in the literature ${ }^{19)}$ that the cellular structures preserved during the transformation of pre-ceramic to ceramic, porous ceramics with an extremely fine and homogenous cell structure, can be fabricated utilizing this process. ${ }^{19)}$ The group also proposed different manufacturing processing of polysiloxane-derived porous ceramics and noted the importance of the direct foaming in another study. ${ }^{20}$

Other methods included the impregnation of a polymer cellular matrix with a ceramic suspension, and subsequent squeezing out, drying, and thermal treatment to remove the organic components were practiced to get better porous ceramics. ${ }^{21)}$ The introduction of ceramic fibers into the mixture, followed by molding with binders and the subsequent thermal treatment of the molded products, can also yield porous materials. ${ }^{22)}$ Uniform, finely cellular foam can be produced by mixing into the ceramic suspension frothing agents that stabilize the resultant three-phase foam. ${ }^{23)}$ Such cellular structures are preserved under subsequent drying and firing. Impregnating a polymeric template increased struts throughout the material and thus increased the strength of the resulting ceramic foams. ${ }^{24)}$ All these previous studies particularly discussed the processing of porous ceramics by direct foaming. The porosity of ceramics produced in this way depends on the content and grain size. Chemical formations of gas bubbles within a ceramic mixture can also increase porosity. These include chemical reactions in the ceramic suspension or the decomposition of gasforming additives. However, the process that is discussed further has higher advantages.

Early twentieth century work by Ramsden ${ }^{25)}$ and Pickering showed that solid particles adsorbed at liquid-liquid interfaces can stabilize the resulting Pickering emulsions ${ }^{26}$ ) through the introduced surface active molecules lowering 
the system's free energy by reducing the liquid-liquid interfacial area. ${ }^{27,28)}$ Utilizing this phenomenon, a new method was proposed with the introduction of air into a colloidal suspension when making highly porous foam ceramics. ${ }^{29-33)}$ It was a great discovery on the processing of porous ceramics by Gauckler's group ${ }^{3)}$ where wet chemical methods were developed to incorporate gaseous phases into ceramic suspensions consisting of ceramic powder, solvent, dispersants, surfactants and gelling agents. ${ }^{34-38)}$ The surfactant films can reduce surface tension, increase surface viscosity and create electrostatic forces to prevent foam from collapsing. The stabilization and de-stabilization mechanisms of coated bubbles exposed to surfactants to produce metallic foams are also discussed elsewhere. ${ }^{39-45)}$ Stabilization of the introduced species' surfaces is required to overcome coalescence, Ostwald ripening and phase separation and can be achieved using lower-energy molecules for droplet formation. These provide steric and electrostatic barriers against coalescence. $^{43,46)}$ The process has been called "direct foaming" and is achieved by the hydrophobization of particles' surfaces; the incorporation of the gaseous phase can result from mechanical frothing, injection of a gas stream, gas-releasing chemical reactions or solvent evaporation. ${ }^{3)}$ Its simplicity, low cost and versatility has made it popular for the manufacture of porous ceramics. Less defective components, as compared with dry processing, have recently been shown to result from the wet processing of powders. It allows better control of the interactions between the powder and the particles and increases the homogeneity of particles packing in the wet stage, which leads to fewer and smaller defects in the final microstructure. This can be achieved either by consolidating the dispersion medium or by flocculating or coagulating the particles in the liquid medium. ${ }^{47-49)}$ As stated previously, ceramics microstructures and properties depend on their fabrication method. Therefore, consideration of the methods' costs, simplicity and versatility is important. Direct foaming produces porous materials by the incorporation of air into a suspension or liquid medium. The foam structure is then set by high-temperature sintering to obtain crack-free, high-strength porous ceramics. The suspensions are stabilized in situ through the hydrophobization of the suspended particles by short chain amphiphilic molecules. The coated, hydrophobic particles irreversibly adsorb onto the air-water interface, thus stabilizing it as shown in Fig. 1. ${ }^{50)}$ Here the processes for the production of the wet foam to porous ceramics is shown achieved by incorporating air into the stabilized suspension, which is subsequently set or sintered to form a structure of air bubbles created to obtain high-strength porous ceramics. These wet foams can remain stable for several days and show no bubble coarsening, drainage or creaming. The short-chain amphiphiles modify in-situ wetting behavior of the particles' surfaces, as in a Pickering emulsion. ${ }^{25)}$ Earlier studies also described that ultra-stable wet foams can be produced by direct foaming using particles instead of surfactants as foam stabilizers. ${ }^{4,31)}$

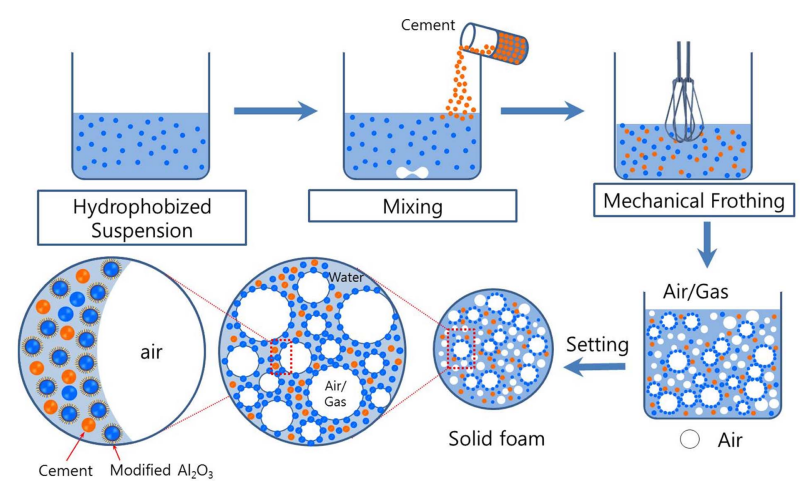

Fig. 1. Process of direct foaming and self-setting, explaining the conditions in the resultant foams. ${ }^{50)}$

This paper explores the stabilization of wet foams by colloidal amphiphilic particles and the development of fabrication techniques of solid macro porous ceramics with tailored microstructures. Given the importance of ceramics' foam microstructures, the effects of foam precursor suspensions bubble size, distribution, contact angle and surface tension - on the resultant porous ceramics' mechanical and physical properties are assessed here. ${ }^{34,40)}$ Control of these parameters allow the tailoring of the microstructures of porous ceramics produced by direct foaming. We deal with easily tailored microstructures without the use of chemical aided drying or a gelling agent for the shaping of the foams. It also describes the successful process with the micro sized particles and includes both the sintering and self-setting porous ceramics produced by the process. In simpler words, we describe simple ways to process porous materials. We also show the process followed for the preparation of self-setting porous ceramics by the process of direct foaming. Cement particles were added in different compositions to the hydrophobized colloidal suspension of $\mathrm{Al}_{2} \mathrm{O}_{3}$, which after mechanical frothing and setting resulted in a porous ceramic structure with closed and open pores. ${ }^{51-53)}$

This literature focuses on describing all the conditions involved, from suspension stability to wet foam stability, leading to the highly stable porous ceramics with tailored microstructures by the process of direct foaming with numerous advantageous characteristics for future applications. Detailed information on the process with the major phenomenon of the processing steps are described thoroughly further in the literature under different headings.

\section{Processing to Direct Foaming}

\subsection{Suspension and In-Situ Hydrophobization}

Colloids are suspensions or liquid foams that are generally thermodynamically unstable. The instability arises due to their high gas-liquid interfacial areas, which raises the free energy of the system. To achieve a stable system, free energy must be minimized. The electro kinetic properties of a colloidal system can be described using the zeta potential as shown in Fig. 2(a). Higher charges on the particles' sur- 


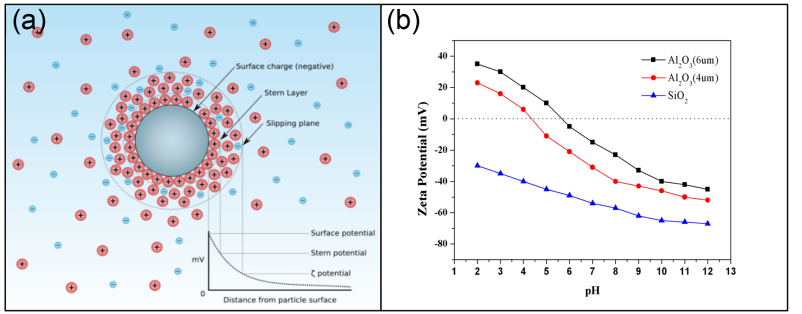

Fig. 2. The distribution of charges in a colloidal suspension: higher charges at the particles surfaces can stabilize the system (a) Zeta potential of raw $\mathrm{Al}_{2} \mathrm{O}_{3}$ and $\mathrm{SiO}_{2}$ colloidal particles (b).

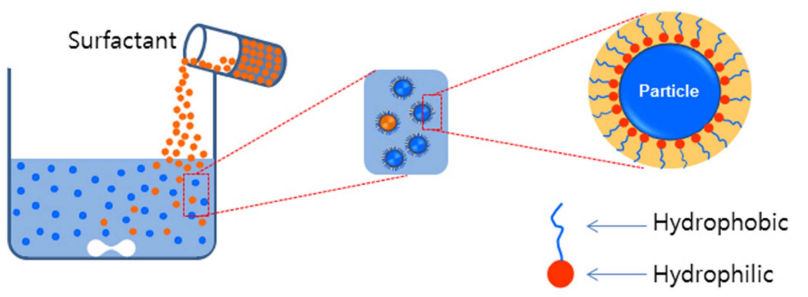

Fig. 3. Process of in-situ hydrophobization.

faces stabilize a colloid by preventing the particles from coming into contact and coalescing. A suspension's $\mathrm{pH}$ affects its charge distribution, and hence its zeta potential. The isoelectric point (IEP) is the $\mathrm{pH}$ at which a colloid's zeta potential is zero; it can be used to derive information about the $\mathrm{pH}$ ranges in which a colloid is stable. A suspension's $\mathrm{pH}$ can be modified to allow a dissociated surfactant to adsorb electrostatically as counter ions onto oppositely charged alumina hydroxyl surface groups. ${ }^{54)}$ The suspension's inorganic particles can be stabilized in situ by the particels' hydrophobization with different colloidal particles containing predominantly $-\mathrm{OH}_{2}^{+},-\mathrm{OH}$ and $-\mathrm{O}^{-}$surface groups. As shown in Fig. 3, short chain amphiphiles with hydrophilic heads and hydrophobic tails were used for the hydrophobization process. Surfaces with predominantly $-\mathrm{OH}_{2}^{+}$, and $-\mathrm{OH}$ groups can be achieved on inorganic alumina particles at $\mathrm{pH} 4.5$ and pH 9.5, respectively.

Fig. 2(b), confirmed the surface exhibiting primary $-\mathrm{OH}_{2}^{+}$ (positive net charge) and - $\mathrm{OH}$ (neutral net charge) groups under those conditions. ${ }^{55,56)}$ The surface hydroxyl groups $\left(-\mathrm{OH}\right.$ or $\left.-\mathrm{OH}_{2}^{+}\right)$were replaced by one or more of the molecule's hydroxyl groups $(-\mathrm{OH}$ or $-\mathrm{O})$. Therefore, the adsorbtion of gallate molecules didn't necessarily require oppositely charged surfaces and amphiphiles that can be used at $\mathrm{pH}$ values at which the surface groups and the molecules exhibit the same charge polarity. Hydrophobizing adsorption can change the wettability of particles at the interface of two immiscible phases and the system is stabilized by the neutral forces between the particles and the amphiphilic coatings. Therefore, the choice of amphiphile depends upon the IEP and the zero net charge of the oxide. Surface hydrophobization can be accomplished by choosing amphiphiles with functional groups that react with the surface hydroxyl groups. Pyro-gallol groups can efficiently adsorb on oxide

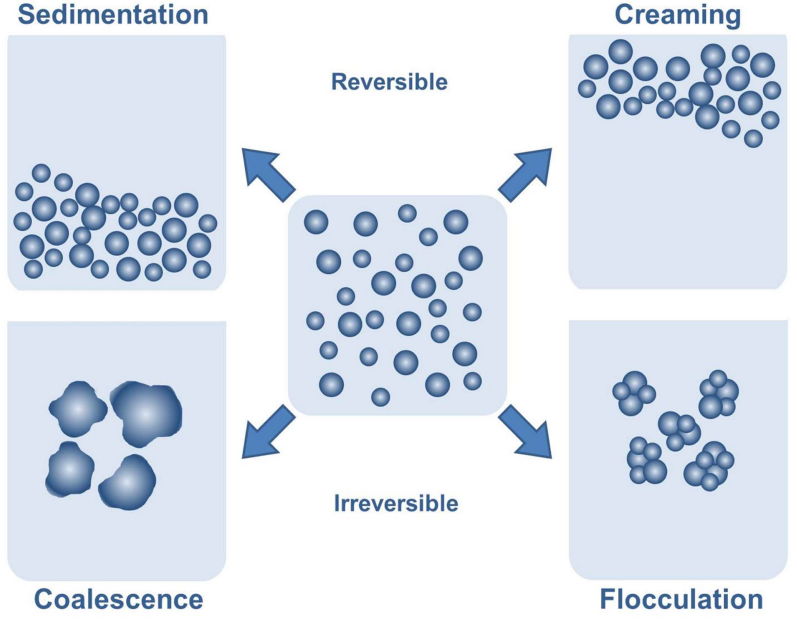

Fig. 4. The destabilization of colloidal suspensions

surfaces via ligand exchange reactions ${ }^{56)}$ and thus can be used with a short hydrocarbon tail to modify the surfaces of particles with intermediate IEPs. Amphiphiles of short chain carboxylic acids and gallates are expected to adsorb well onto alumina particles.

As short chain molecules are highly soluble in water, they can hydrophobize a larger number of particles and stabilize large interfacial areas. Propyl gallate has been used to modify the surfaces of particles by ligand exchange reactions. ${ }^{34,56)}$ The selection of amphiphiles with suitable head groups and tail lengths allow the surface hydrophobization of particles of various compositions.

\subsection{Destabilization in a Suspension}

Colloidal dispersions can be thermodynamically unstable, with long-term kinetic stability determining their shelf-life. The main destabilization mechanisms are drainage (creaming and sedimentation), coalescence and flocculation, as shown in Fig. 4. Creaming and sedimentation are caused by gravity: lighter particles float and heavier particles settle. They are reversible when mechanically agitated (homogenization or simple shaking), which will re-disperse the suspension. Coalescence and flocculation are not reversible and so affect a suspension's stability. Flocculation is the clustering of colloidal particles via attractive van der Waals forces. It can be overcome or prevented by higher-energy ultra-sonification or by generating particles with repulsive interactions. ${ }^{27,28)}$

Coalescence is the greatest destabilizing mechanism. It involves smaller particles collapsing into each other, forming larger particles with different properties. Many dispersion techniques have been developed to prevent coalescence. ${ }^{57)}$ Other procedures of destabilization also occur: When the interaction potential between the particles is purely attractive, the aggregation process is solely limited by mutual diffusion (or Brownian motion) of the particles. This should be overcome by the surface charge, which overlaps between the double layers of two approaching particles, resulting in 
repulsive interaction potential, which leads to stabilization. The stabilization of the colloidal suspension in our research has been discussed.

\subsection{Suspension Stability}

The formation of the foams requires the adsorption of particles on the surfaces of air bubbles upon their formation. Alumina particles can be hydrophobized by modification with short-chain carboxylic acids: the carboxylate groups adsorb onto the alumina's surface, ${ }^{56,58)}$ leaving the hydrophobic tail in contact with the aqueous solution. This has shown stabilization of dispersions. ${ }^{59,60)}$ The hydrophobicity imparted by the first layer of de-pronated amphiphiles adsorbed onto the surface, which led to an energetically unfavorable exposure of hydrophobic species to the aqueous phase. This favors the adsorption of additional molecules from the aqueous phase onto the particles' surfaces to decrease the system's free energy, which determines the stability of a suspension or wet foam. Particles attached to foam and mist gas-liquid interfaces lower the overall free energy by replacing part of the interfacial area rather than reducing the interfacial tension, as in the case of surfactants. ${ }^{2}$ The energy of the attachment, i.e, the Gibbs free energy (G), gained by the adsorption of a particle of radius $r$ at the interface, can be calculated using simple geometrical arguments that lead to the following equation.

$$
\mathrm{G}=\pi \mathrm{r}^{2} \gamma_{\mathrm{LG}}(1-\cos \theta) \text { for } \theta<90^{\circ}
$$

where $\theta$ is the contact angle, $\gamma_{\mathrm{LG}}$ is the interfacial tension between the liquid and air interface, and $r$ is the radius of the particles.

While the maximum energy gain can only be achieved at $\theta=90^{\circ}$, contact angles as low as $20^{\circ}$ can yield attachment energies in the order of $10^{3} \mathrm{kT}$ in systems of $100 \mathrm{~nm}$ particles. $^{2)}$ The high energy associated with the adsorption of particles at interfaces contrasts to low adsorption energies of surfactants and leads to foams stabilized by particles being more stable than those stabilized with surfactants. It also leads to steric layers that strongly hinder bubbles' shrinkage and expansion, thus minimizing Ostwald ripening for very long periods of time. ${ }^{30}$ The particle systems in the experiments in our laboratory had adsorption achieved by ligand exchange, whereby a surface hydroxyl group is exchanged for another group. This occurred because of the favorable change in the surface charge by the removal of $\left(\mathrm{OH}_{2}^{+}\right)$, a better leaving group, and replacement with $(-\mathrm{OH}) .{ }^{49)}$

\subsection{Contact Angle and Surface Tension}

After the stabilizing effects of zeta potential and $\mathrm{pH}$, the contact angle and surface tension are important determinants of the properties of colloidal systems. Once a suspension is stabilized, the degree of hydrophobization is the main property that affects the production of foam. Giving credit to their thermodynamic instability, foams are often kinetically stabilized through the adsorption of surface active molecules or colloidal particles at the gas-liquid interfaces. ${ }^{33,60)}$ The adsorbed molecules and particles stabilize the system by inhibiting the coalescence and Ostwald ripening of droplets and bubbles. Adsorption at the fluid interfaces occurs when particles are not completely wetted by any of the fluids, thus exhibiting a finite equilibrium contact angle at the triple phase boundary. The equilibrium contact angle $(\theta)$ is determined by the balancing of the interfacial tensions. A decrease in surface tension with an increase in the initial amphiphile concentration was observed for all the evaluated suspensions. However, above a critical amphiphile concentration, surface tension decreased sharply. Above this critical amphiphile concentration, the particles are sufficiently hydrophobic at the air-water interface and decrease greater surface tension than that expected from free amphiphiles alone. ${ }^{61)}$ This significant reduction in surface tension upon particle adsorption was caused by a decrease in the total area of the highly energetic air-water interface. Similar surface tension effects have been observed in systems employing various amphiphiles. ${ }^{61)}$

Controlling particles' contact angles at the interface is important as it determines their wettability or the degree of hydrophobization as shown in Fig. 5. Tailoring the contact angles of particles via modification of chemical composition enables the creation of foams with a variety of functionalities. ${ }^{61)}$ The contact angle depends on surface chemistry, roughness, impurities, particle size and the composition of the fluid phases. Theoretical and experimental work has shown that stabilization is achieved when contact angles are in an intermediate range of $20^{\circ}-86^{\circ}$ for oil-in-water foams and of $94^{\circ}-160^{\circ}$ for water in oil mists. The contact angle can also be tailored by changing the surface chemistry of the particles or adjusting the composition of the fluids. Metallic and ceramic particles can achieve any contact angle $\left(0<\theta<180^{\circ}\right)$ by reacting or adsorbing hydrophobic molecules on their surfaces. The use of short amphiphiles to tailor particles wettability is a general and versatile approach for the surface modification of a wide range of ceramic and metallic materials. ${ }^{2,56,61)}$

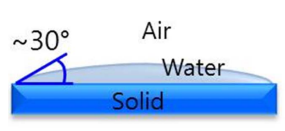

Hydrophilic

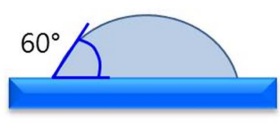

Partially

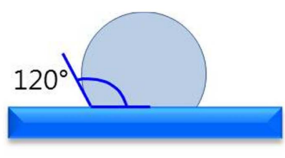

Partially hydrophobic

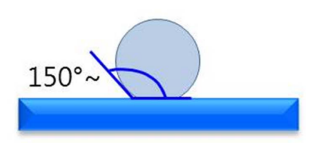

Hydrophobic

Fig. 5. Degree of hydrophobization of particles demonstrated with the corresponding contact angle of the initial suspension. 


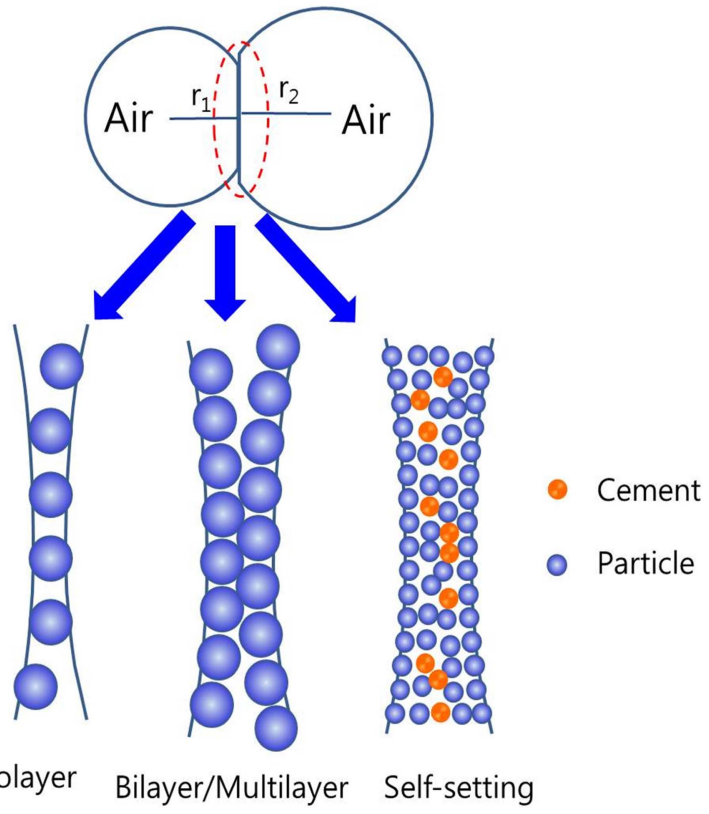

Fig. 6. Bubble interface and thin films of wet foams.

\subsection{Wet Foam Stability}

Liquid foams are thermodynamically unstable due to their high gas-liquid interfacial area. Several physical processes can occur to decrease the overall free energy and destabilize the foam. ${ }^{58-60)}$ Drainage occurs through gravity; light gas bubbles rise forming a denser foam layer, while the heavier liquid phase is concentrated below. Coalescence takes place when the thin films formed after drainage is not stable enough to keep adjacent cells apart. Their collapse results in the joining of neighboring bubbles. The stability of the thin films is shown in Fig. 6, and is described in terms of attractive and repulsive interactions between the bubbles.

There are single or bilayers of modified and unmodified particles on the films that maintain stability of the films, thus withstanding the change in the pressure between the bubbles. The Van der Waal's force can be overcome by electrostatic forces, steric repulsions force or by ligand exchange reactions. Surfactant or particles adsorbed at the air-water interface can also reduce Van der Waal's forces. ${ }^{46,60)}$ Ostwald ripening or disproportionation is another destabilizing effect that is more difficult to overcome. It occurs due to differences in the Laplace pressures between bubbles of different sizes. Laplace pressure inside a gas bubble arises from the curvature of the air-water interface. For spherical bubbles of radius $R$ and gas-liquid interfacial energy $\gamma$, the Laplace pressure $\mathrm{P}$ is given by $2 \gamma / \mathrm{R}^{46,48}$ ) The difference in the Laplace pressure between bubbles of distinct sizes (R) leads to bubble disproportionation and Ostwald ripening because of the steady diffusion of gas molecules from smaller to larger bubbles over time. The pressure and force generated for the stabilization can be also calculated through the measurement of bubbles at the intersection. It can be calculated by the equation given below.

$$
\Delta P=\gamma\left(\frac{1}{R_{1}}+\frac{1}{R_{2}}\right)
$$

where $\Delta \mathrm{P}$ is the Laplace pressure, $\gamma$ the interfacial tension, and $R_{1}$ and $R_{2}$ are radii of interfacing bubbles.

These processes can be slowed by using surfactants or particles adsorbed at the interface, which decrease the interfacial energy. Wet foam's stability is also related to the degree of hydrophobicity achieved from the surfactant, which replaces part of the highly energetic interface area and lowers the free energy of the system, leading to an apparent reduction in the surface tension of the suspension. ${ }^{61)}$ Stability also depends on surface charge screening, the electrical diffuse layer around a particle's surface not sufficiently thick to overcome the attractive Van der Waal's forces between particles. Overcoming the Van der Waal's attractions requires a stable hydrophobizing mechanism (examined above). These actions' combined effects may collapse the foam within minutes after air incorporation. The life time of foams have been increased from several hours to days and months by the adsorption of the short chain amphiphilic molecules ${ }^{38,40,41)}$ while only a few minutes or hours' stabilization results from the use of long-chain surfactants or proteins at the air-water interface. Unlike other particle-stabilized foams ${ }^{2}$ these foams percolate throughout the whole liquid phase and exhibit no drainage over days and months due to the high concentration of modified particles in the initial suspension, which allows for the stabilization of very large total air-water interfacial areas.

\section{Processing and Microstructure of Sintered and Self-Setting Porous Ceramics}

The colloidal suspensions were prepared using propyl gallate as an amphiphile through the stepwise addition of alumina powder to an aqueous mixture of $0.01 \mathrm{M} \mathrm{NaOH}$ and $0.01 \mathrm{M}$ propyl gallate. The solid loadings and $\mathrm{pH}$ of the suspensions were initially fixed at $50 \mathrm{vol} \%$ and 9.9 , respectively. Homogenization and de-agglomeration were performed via ball milling for at least $24 \mathrm{~h}$ using polyethylene milling pots and alumina balls (10 $\mathrm{mm}$ in diameter - 2:1 ratio of balls to powder). The propyl gallate was adjusted to the required concentration in the final suspension (1-2 wt\% to $\left.\mathrm{Al}_{2} \mathrm{O}_{3}\right)$. This was dissolved in $\mathrm{NaOH}(\mathrm{pH}>10)$ and added dropwise to the ball milled suspension under slight stirring in order to avoid local particle agglomeration. Finally, the $\mathrm{pH}$ was set at 9.9 and water was added until the solid content was $30 \mathrm{vol} \%$. In other experiments, suspensions under various $\mathrm{pH}$ values were also tested. The $\mathrm{SiO}_{2}$ suspensions were prepared through the addition of dry $\mathrm{SiO}_{2}$ powder to water in order to achieve solid loadings of $45 \mathrm{vol} \%$. The $\mathrm{SiO}_{2}$ was unmodified and no further $\mathrm{pH}$ modification was required. The suspensions were then de-agglomerated and homogenized via ball milling. Through stirring, the $\mathrm{SiO}_{2}$ suspension was added drop wise in different ratios to the alu- 


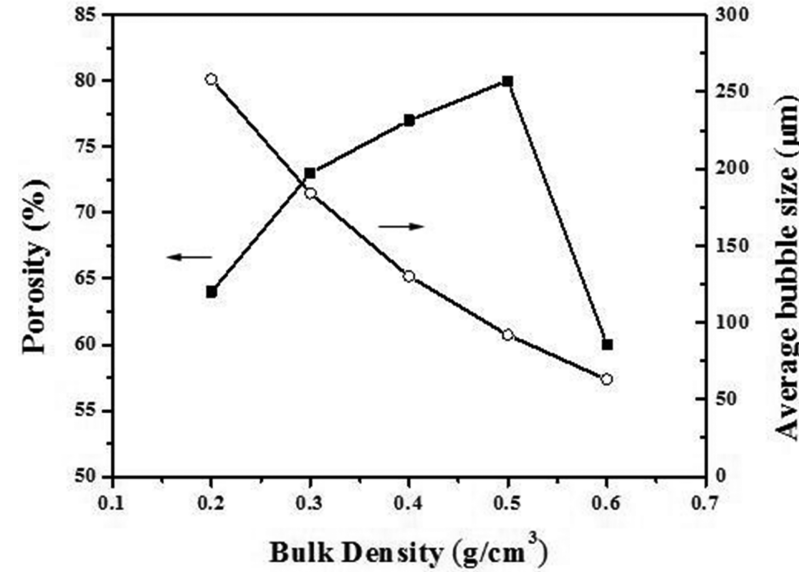

Fig. 7. Porosity and average bubble size of $\mathrm{Al}_{2} \mathrm{O}_{3}-\mathrm{SiO}_{2}$ ceramics with respect to density after sintering at $1300^{\circ} \mathrm{C}$.

mina suspensions containing propyl gallate. The $\mathrm{pH}$ was set at 4.5 to 9.9 ; that was different from other tests. The total solid content of the mixture was fixed at $30 \mathrm{vol} \%$. Various blends of modified and unmodified particles were prepared and tested with a total solid content of $30 \mathrm{vol} \%$ under varying $\mathrm{pH}$ conditions.

The effects of amphiphile strength on contact angle and wet foam stability were assessed and can be seen in our publication. ${ }^{34,38)}$ There was a change in the foam stability and its corresponding contact angles too. The mixture of modified and unmodified particles showed similar properties with increasing particle concentration, with the only exception being the destabilization of suspensions with high silica content. Ratios of alumina to silica of 0.1- 0.5 resulted in stable foams with high porosity ${ }^{40)}$ The decrease of air content with solids content over $50 \mathrm{vol} \%$ was further removed due to the increased viscosities of the initial suspensions. Further increase of particle concentration increased viscosity, hindering the incorporation of air for foam formation.

The required partial hydrophobization of the particles occurs at this point which leads to porous ceramics with higher porosity. These foams had linear shrinkage of less than $25 \%$ after sintering and larger surface areas, and gained a porosity of about $80 \%$ after sintering. ${ }^{34)}$ This also explains how the increase in contact angle and decrease in surface tension leads to average bubbles of less than $100 \mu \mathrm{m}$ with higher porosity and mechanical strength. These are only the results of our experiments with certain parameters, and the results may vary with use of different stabilizers that gives different level of particle hydrophobization and shows the areas of stabile and unstable zones. The properties of the sintered ceramics, bulk density and compressive strength, are shown in Fig. 7. Porosity and bubble size increase with the slight increase in the density, but after reaching a maximum of $0.5 \mathrm{~g} / \mathrm{cm}^{3}$, it decreases rapidly.

Fig. 8 shows that the compressive strengths of the macro porous ceramics increased with an increasing amphiphile

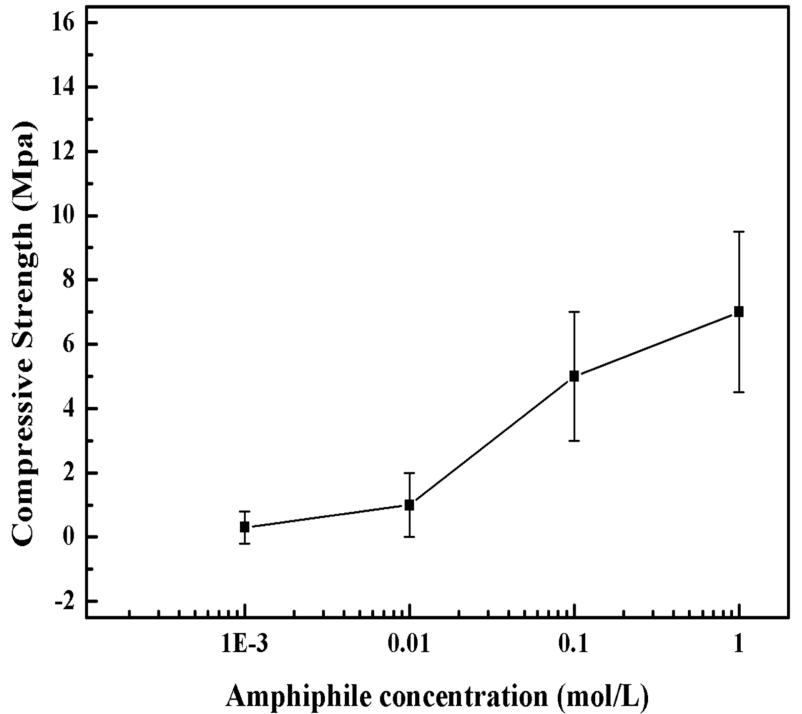

Fig. 8. Compressive strength of porous $\mathrm{Al}_{2} \mathrm{O}_{3}-\mathrm{SiO}_{2}$ ceramics with respect to amphiphile concentration.

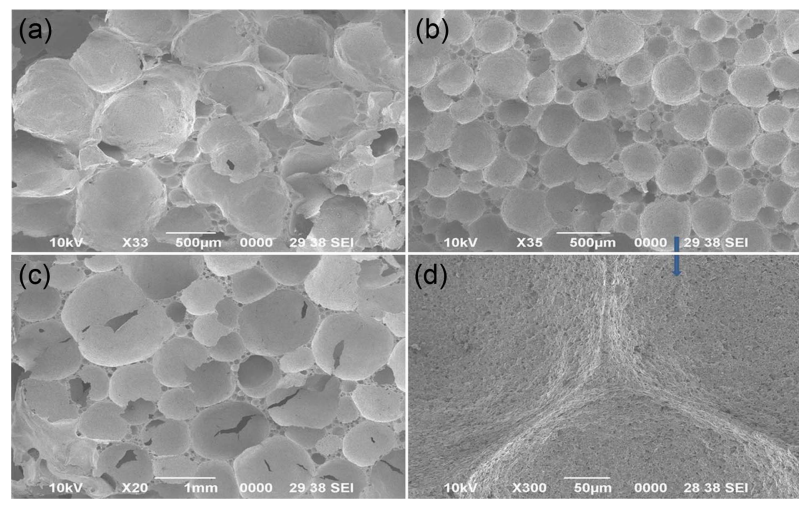

Fig. 9. Microstructures of foams sintered at $1300^{\circ} \mathrm{C}$, with $30 \mathrm{vol} \%$ total solid content, $0.01 \mathrm{M}$ propyl gallate, and different ratios of $\mathrm{SiO}_{2}$ content: a) $\mathrm{Al}_{2} \mathrm{O}_{3}$, b) $1: 0.25$, c) $1: 0.5$, and d) Intra cellular structures. ${ }^{40)}$

content due to greater adsorption of particles. It increases the density of the sintered ceramics with lesser air content of the foam. Higher amphiphile content led to smaller average bubble sizes and less porosity, which also proves the increasing compressive strength of the sintered ceramics. Less content of the amphiphiles gave weak foams due to a lager bubble size and less density, whereas with higher concentration of amphiphile gave smaller, compactly distributed bubbles, that on sintering gave extra strength to the structure.

Fig. 9 shows the microstructure obtained from the alumina at similar molar concentrations regarding the amphiphiles but with different ratios of the $\mathrm{SiO}_{2}$ added to the suspension. Here, we observe no difference in the molecular structure but the pore size increases with the increase in the additives in the form of $\mathrm{SiO}_{2}$. It also shows us that the inter pore differences were highly distinguished and uniform in the samples with $\mathrm{SiO}_{2}$ content with the ratio of $1: 0.25$ and $1: 0.5$ in the initial suspension before a foaming increase in 

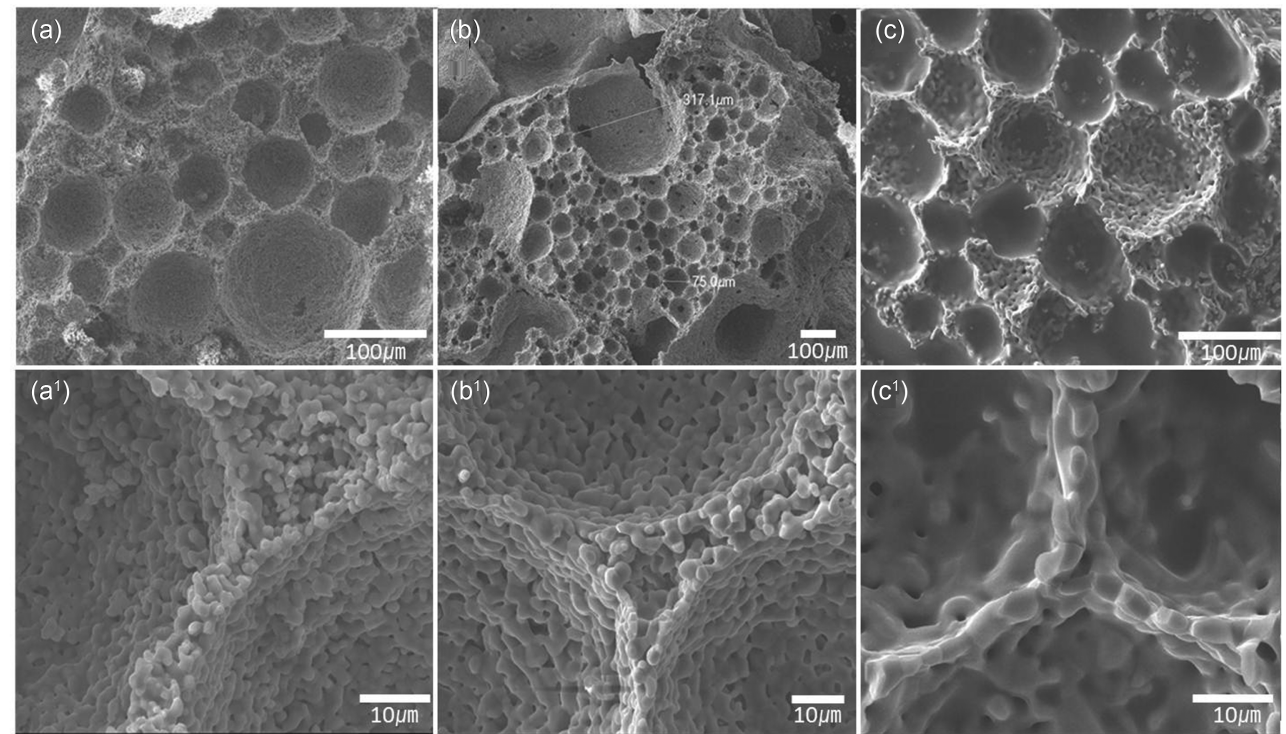

Fig. 10. Microstructures of sintered foams: a) and $a^{1)}(15$ vol\% H.A+30 vol\% $\beta$-TCP at $1: 1)$, b) and $b^{1)}(15$ vol\% H.A+35 vol\% $\beta$-TCP at $1: 1)$, at $1200^{\circ} \mathrm{C}$, and $\left.\mathrm{c}\right)$ and $\mathrm{c}^{1)}\left(15 \mathrm{vol} \% \mathrm{H} . \mathrm{A}+30 \mathrm{vol} \% \beta\right.$-TCP at $1: 1$ at $\left.\left.1300^{\circ} \mathrm{C}\right){ }^{53}\right)$

the ratio of additives resulted in larger pore size but less porosity due to the increase of struts and also led to higher instability of the wet foams resulting in unstable porous ceramics.

Fig. 10 shows porous hydroxyl apatite sintered foams with different amount of additives. ${ }^{53)}$ The microstructures were tailored by adding the additive as non-modified $\beta$ TCP suspension at a ratio of $1: 1$ with a stabilized hydroxyapatite suspension. The sintered structure showed smaller pores and the formed structure showed higher mechanical strength due to its homogenous distribution. To produce better results, we adjusted the volumetric composition as already defined in the literature; the microstructures were achieved as shown in Fig. 10. The structures in Figs. 10(a) and $\left(a^{1}\right)$ and (b) and $\left(b^{1}\right)$ show the uniform pore distribution with an average pore size of less than $100 \mu \mathrm{m}$. The average intercellular differences were 1 to $5 \mu \mathrm{m}$ and were uniform. The sintered ceramics also showed a linear shrinkage of less than $10 \%$ with higher mechanical strength. To confirm our results, the best sample was repeated with the same procedures but with an increased sintering temperature. The microstructures are shown in Figs. 10(c) and $\left(c^{1}\right)$ the increased temperature resulted in melted structured bio ceramics, as hydroxyapatite had a lower melting point.

We also performed experiments on the self-setting wet foams to porous ceramics by direct foaming. ${ }^{50)}$ As propyl gallate plays a role in retarding the rate of the setting, we decided to use an accelerator to the setting of the cement in the form of $\mathrm{LiCO}_{3}$ which increased the rate of the reaction. ${ }^{51,52)}$ Introducing lithium ions, tetrahedral symmetry with the aluminum hydroxide is formed, which lowers the overall activation energy for forming the different hydration products. The change in the setting time of the wet foam was reported with respect to the amount of cement and a

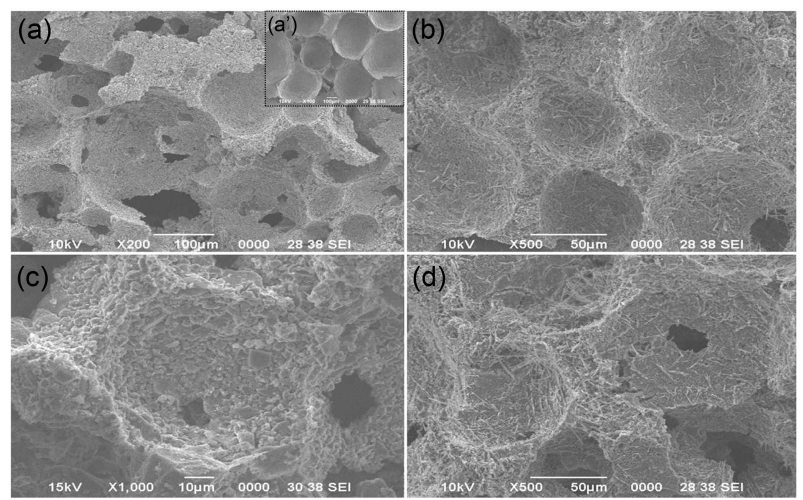

Fig. 11. Microstructures of self-setting wet foams with $15 \mathrm{vol} \%$ $\mathrm{Al}_{2} \mathrm{O}_{3}+10 \mathrm{wt} \% \mathrm{LiCO}_{3}$ to cement: a) $20 \mathrm{vol} \%$ cement room dried, a') sintered $\mathrm{Al}_{2} \mathrm{O}_{3}-\mathrm{SiO}_{2}$ ceramics at 1300 for $1 \mathrm{~h}, \mathrm{~b}) 20$ vol\% cement humid dried at $35^{\circ} \mathrm{C}$, c) 25 vol\% cement room dried, and d) 25 vol\% cement humid dried at $35^{\circ} \mathrm{C}{ }^{50)}$

different amount of accelerator. ${ }^{50)}$ It showed rapid setting of the foams with cement of $25 \mathrm{vol} \%$ to $\mathrm{Al}_{2} \mathrm{O}_{3}$ before foaming. However, the continuous addition of the accelerator of more than $15 \mathrm{wt} \%$ to the weight of cement led to destabilization and the formation of a liquid-like structure, due to the presence of a higher amount of reactive $\mathrm{Li}+$ ions. ${ }^{51)}$ These experiments also revealed the fact that an increase in the accelerator helped increase the setting time but decreased the long term strength of the samples. Fig. 11 shows the porous structures of the self-setting ceramics formed by the process of direct foaming described in the literature. ${ }^{51,52)}$ The focus of the research was to increase this property, which was achieved by adding the unmodified $\mathrm{SiO}_{2}$ suspension in the suspension in different ratios, compositions and setting of the foams in different environmental conditions. ${ }^{50)}$ 


\section{Conclusions}

All continuous microstructures of both sintered and nonsintered porous ceramics open new prospects for invention in cellular ceramics and ongoing research is upgrading the porous structures and its properties for diverse applications in our everyday lives. The microstructures and properties of porous ceramics are affected by their method of synthesis. Direct foaming can simply, inexpensively and quickly prepare macro porous ceramics. Open or closed porosities of 45\%-85\% have been demonstrated. Cellular structures prepared by direct foaming are generally stronger than those prepared by replica synthesis due mainly to the absence of flaws in the cell struts. Given the importance of the chosen synthetic method, this review explains the processing of currently available processes for forming porous ceramics. Its continuous study will result in further improvements in the methods and wider applicability of its products. The pores produced by this method resulted from the direct incorporation of air bubbles into a ceramic suspension, eliminating the need for pyrolysis before sintering. The leading processing steps, such as controlled evaporation, chemical drying, and removal of templates or pyrolysis are removed. Porous ceramics prepared by this process gives us advantages over excessive use of energy and additional chemicals for the production and processing of cellular ceramics. Conclusively we can say that it makes the processing steps easy to proceed for industrial production.

\section{Acknowledgment}

This research was financially supported by the Ministry of Education, Science Technology (MEST), and the National Research Foundation of Korea (NRF) through the Human Resource Training Project for Regional Innovation, and the Ministry of Knowledge Economy (MKE) and the Korea Institute for Advancement of Technology (KIAT) through the Regional Innovation System.

\section{REFERENCES}

1. M. Scheffler and P. Colombo, "Cellular Ceramics: Structure, Manufacturing, Properties and Applications," p. 645, Weinheim, Wiley-VCH, Verlag GmbH \& Co. KGaA, 2005.

2. A. R. Studart, U. T. Gonzenbach, E. Tervoort, and L. J. Gauckler, "Processing Routes to Macroporous Ceramics - A Review," J. Am. Ceram. Soc., 89 [6] 1771-89 (2006).

3. L. J. Gauckler, A. Studart, E. Tervoort, U. T. Gonzanbech, and I. Akartuna, "Ultrastable Particle Stabilized Foams and Emulsions," U.S. Pat No:A1 0325780, (Dec. 31, 2009).

4. Y. Guzman, "Certain Principles of Formation of Porous Ceramic Structures. Properties and Applications-A Review," Glass Ceram., 9 28-31 (2003).

5. P. Colombo, "In Praise of Pores," Science, 32 381-83 (2008).

6. P. Colombo and J .R. Hellmann, "Ceramic Foams from PreCeramic Polymers," Mater. Res. Innovat., 6 260-72 (2002).

7. P. Colombo and E. Bernardo, "Macro- and Micro-cellular
Porous Ceramics from Preceramic Polymers," Compos. Sci. Technol., 63 2353-59 (2003).

8. P. Colombo, "Conventional and Novel Processing Methods for Cellular Ceramics," Phil. Trans. R. Soc. A., 364 109-24 (2006).

9. P. Colombo, "Engineering Porosity in Polymer-Derived Ceramics," J. Eur. Ceram. Soc., 28 1389-95 (2008).

10. P. Greil, "Advanced Engineering Ceramics," Adv. Mater., 14 [10] 709-16 (2002).

11. J. Zeschky, F. G. Neunhoeffer, J. Neubauer, S. H. J. Lo, B. Kummer, M. Scheffler, and P. Greil, "Preceramic Polymer Derived Ceramics," Compos. Sci. Technol., 63 2361-70 (2003).

12. J. Banhart, "Manufacturing Routes for Metallic foams," JOM 52[12] 22-27 (2000).

13. J. Banhart, "Manufacture, Classification and application of Cellular Metals and Foams," Prog. Mater Sci., 46 559-632 (2001).

14. F. V. Zeppelin, M. Hirscher, H. Stanzick, and J. Banhart, "Desorbtion of Hydrogen from Blowing Agents Used for Foaming Metals," Compos. Sci. Technol., 63 2293-300 (2003).

15. C. Tuck and J. R. Evans, "Porous Ceramics from Aqueous Foams," J. Mater. Sci. Lett., 18 1003-05 (1999).

16. H. X. Peng, Z. Fan, J. R. G. Evans, and J. J. Busfield, "Microstructure of Ceramic Foams," J. Eur. Ceram. Soc., 20 807-13 (2000)

17. J. R. G. Evans, "Seventy Ways to Make Porous Ceramics," J. Eur. Ceram. Soc., 28 1421-32 (2008).

18. Y. W. Kim, S. H. Kim, C. Wang, and C. H. Park, "Fabrication of Microcellular Ceramics Using Gaseous Carbon Dioxide," J. Am. Ceram. Soc., 86 [12] 2231-33 (2003).

19. Y. W. Kim and C. B. Park, "Processing of Microcellular Preceramics Using Carbon Dioxide," Compos. Sci. Technol., 63 2371-77 (2003).

20. B. V. Manoj Kumar and Y. W. Kim, "Processing of Polysiloxane-Derived Porous Ceramics: Tropical Review," Sci. Technol. Adv. Mater., 11 1-16 (2010).

21. O. Lyckfeldt and J. M. Ferreira, "Processing of Porous Ceramics by Starch Consolidation," J. Eur. Ceram. Soc., 28 131-40 (1998).

22. F. Schuth and W. Schmidt, "Microporous and Mesoporous Materials," Adv. Eng. Mater., 4 [5] 269-79 (2005).

23. S. Barg, C. Soltmann, M. Andrade, D. Koch, and G. Grathwohl, "Cellular Ceramics by Direct foaming of Emulsified Ceramic Powder Suspensions," J. Am. Ceram. Soc., 91 [9] 2823-29 (2008).

24. B. Neirinck, J. Fransaer, O. V. der Biest, and J. Vleugels, "A Novel Route to Produce Porous Ceramics," J. Eur. Ceram. Soc., 29 833-36 (2009).

25. W. Ramsden, "Separation of Solids in the Surface-Layers of Solutions and Suspensions," Proc. R. Soc. London., 72 15664 (1903).

26. S. U. Pickering, "Pickering: Emulsions," J. Chem. Soc., Trans., 91 2001-21(1907).

27. L. J. Gauckler, T. Graule and F. Baader, "Ceramic Forming Using Enzyme Catalyzed Reactions," Mater. Chem. Phys., 61 78-102 (1999).

28. P. C. Hidber, T. J. Graule, and L. J. Gauckler, "Influence of 
the Disperant Structure on Properties of Electrostatically Stabilized Aqueous Alumina Suspension," J. Eur. Ceram. Soc., 17 [2-3] 239-49 (2002).

29. U. T. Gonzenabach, A. R Studart, E. Tervoort, and L. J. Gauckler, "Stabilization of Foams with Inorganic Colloidal Particles," Langmuir., 22 10983-88 (2006).

30. U. T. Gonzenabach, A. R Studart, E. Tervoort, and L. J. Gauckler, "Ultra-Stable Particle-Stabilized Foams," Angew. Chem. Int. Ed., 45 3526-30 (2006).

31. A. R. Studart, U. T. Gonzenbach, I. Akartuna, E. Tervoort, and L. J. Gauckler, "Materials from Foams and Emulsions Stabilized by Colloidal Particles," J. Mater. Chem., 17 3283-89 (2007).

32. U. T. Gonzenbach, A. R. Studart, D. Steinlin, E. Tervoort, and L. J. Gauckler, "Processing of Particle-Stabilized Wet Foams into Porous Ceramics," J. Am. Ceram. Soc., 90 [11] 3407-14 (2007).

33. U. T. Gonzenbach, A. R. Studart, E. Tervoort, and L. J. Gauckler, "Tailoring the Microstruct -ure of Particle-Stabilized Wet Foams," Langmuir., 23 [3] 1025-32 (2007).

34. A. Pokhrel, J. G. Park, W. Zhao, and I. J. Kim, "Functional Porous Ceramics Using Amphiphilic Molecule," J. Ceram. Proc. Res., 13 [4] 420-24 (2012).

35. A. Pokhrel, Zhao Wei, and I. J. Kim, "Wet Foam Stabilized by Amphiphiles to Tailor the Microstructures of Porous Ceramics," Key Eng. Mater., 512-515 288-92(2012).

36. U. T. Gonzenbach, A. R. Studart, E. Tervoort, and L. J. Gauckler, "Macroporous Ceramics from Particle-Stabilized Wet Foams," J. Am. Ceram. Soc., 90 [1] 19-22 (2007).

37. A. Pokhrel, J. G. Park, J. S. Nam, D. S. Cheong, and I. J. Kim, "Stabilization of Wet Foams for Porous Ceramics Using Amphiphilic Particles,” J. Kor. Ceram. Soc., 48 [5] 463-66 (2011)

38. A. Pokhrel, J. G. Park, G. H. Jho, J. Y. Kim, and I. J. Kim, "Controlling the Porosity of Particle Stabilized $\mathrm{Al}_{2} \mathrm{O}_{3}$ Based Ceramics," J. Kor. Ceram. Soc., 48 [6] 600-03 (2011).

39. I. Akartuna, A. R. Studart, E. Tervoot, U. T. Gonzenbach, and L. J. Gauckler, "Stabilization of Oil-in-Water Emulsions by Colloidal Particles Modified with Short Amphiphiles," Langmuir., 24 7161-68 (2008).

40. A. Pokhrel, J. G. Park, S. M. Park, and I. J. Kim, "Tailoring the Microstructure of $\mathrm{Al}_{2} \mathrm{O}_{3}-\mathrm{SiO}_{2}$ Wet Foams to Porous Ceramics," Submitted to J. Ceram. Pro. Res (2012).

41. I. Akartuna, A. R. Studart, E. Tervoot, and L. J. Gauckler, "Macro Porous Ceramics from Particle-Stabilized Emulsions," Adv. Mater., 20 4714-18(2008).

42. I. Akartuna, E. Tervoot, A. R. Studart, and L. J. Gauckler, "General Route for the Assembly of Functional Inorganic Capsules," Langmuir., 25 [21] 12419-24(2009).

43. H. M. Princen and A. D. Kiss, "Rheology of Foams and Highly Concentrated Emulsions," J. Collid Interface Sci., 128 [1] 176 - 87 (1989).

44. B. P. Binks, "Particles as Surfactants-Similarities and Differences," Curr. Opin. Colloid. Interface Sci., 7 21-41 (2002).

45. B. S. Murray, "Stabilization of Bubbles and Foams," Curr.
Opin. Colloid. Interface Sci., 12 231-41 (2007).

46. T. S. Horozov, "Foams and Foam Films Stabilized by Solid Particles," Curr. Opin. Colloid. Interface Sci., 13 134-40 (2008).

47. O. Lyckfeldt and J. M. F. Ferreira, "Processing of Porous Ceramics by Starch Consolidation," J. Eur. Ceram. Soc., 18 131-40 (1998).

48. T. N. Hunter, R. J. Pugh, G. V. Fanks, and G. J. Jameson, "A Role of Particles in Stabilizing Foams and Emulsions," Adv. Colloid. Interface Sci., 13 [7] 57-81 (2008).

49. P. J .Wilde, "Interface: Their Role in Foam and Emulsion Behavior," Curr. Opin. Colloid. Interface Sci., 5 176-81 (2000).

50. J. G. Park, A. Pokhrel S. D. Nam, W. Zhao, B. Basnet, and I. J. Kim, "Self-Setting Wet Foams to Porous Ceramics by Direct Foaming," submitted to Adv. Appl. Ceram. (2012).

51. F. K. Juillerat, U. T. Gonzanbech, P. Elser, A. R. Studart, and L. J. Gauckler, "Microstructural Control of Self-Setting Particle Stabilized Ceramic Foams," J. Am. Ceram. Soc., 94 [1] 77-83 (2011).

52. F. K. Juillerat, U. T. Gonzanbech, A. R. Studart, and L. J. Gauckler, "Self-Setting Particle Stabilized foams with Hierarchical Pore Strucutres," Mater. Lett., 64 1468-70 (2010).

53. A. Pokhrel S. D. Nam, G. H. Cho, and I. J. Kim, "Inorganic Phosphate Wet Foam Stabilization to Porous ceramics by Direct Foaming," submitted to Asian J. Chem. (2012).

54. G. Morris, M. R. Pursell, S. J. Neethling, and J. J. Cilliers, "The Effect of Particle Hydrophobicity, Separation distance and Packing Patterns on the Stability of a Thin Film," J. Colloid Interface Sci., 327 138-44 (2008).

55. N. D. Denkov, I. B. Ivanov, P. A. Kralchevsky, and D. T. Wasan, "A Possible Mechanism of Stabilization of Emulsions by Solid Particles," J. Colloid Interface Sci., 150 [2] 589-93 (1992).

56. D. M. -Alguacil, E. Tervoort, C. Cattin and L. J. Gauckler, "Contact Angle and Adsorbtion Behavior of Carboxylic Acids on $\alpha-\mathrm{Al}_{2} \mathrm{O}_{3}$ Surfaces," J. Colloid Interface Sci., 353 512-18 (2011).

57. G. Kaptay, "On the Equation of the Maximum Capillary Pressure Induced by Solid Particles to Stabilize Emulsions and Foams and on the Emulsion Stability Diagrams," Colloids Surf A., 282-283 387-401 (2006).

58. I. Aranberri, B. P. Binks, J. H. Clint, and P. D. I. Fletcher, "Synthesis of Macroporous Silica from Solid-Stabilized Emulsion Templates," J. Porous Mater., 16 429-37 (2009).

59. E. Dickinson, R. Ettelaie, T. Kostakis, and B. S. Murray, "Factors Controlling the Formation and Stability of Air Bubbles Stabilized by Partially Hydrophobic Silica Nanoparticles," Langmuir., 20 8517-25(2004).

60. B. S. Murray and Ettelaie, "Foam Atability: Proteins and Nanoparticles," Curr. Opin Colloid. Interface Sci., 9314 20 (2004).

61. A. R. Studart, R. Libanori, A. Moreno, U. T. Gonzenbach, E. Tervoort, and L .J. Gauckler, "Unifying model for the Electro Kinetic and Phase Behavior of Aqueous Suspensions Containing Short and Long Amphiphiles," Langmuir., 2711835 -44 (2011). 